\title{
Collimation testing of laser beams having different diameters using compact holographic lateral shearing interferometer (Erratum)
}

\author{
Rajiv Dubey $^{\mathrm{a}, \mathrm{b}}$ and Raj Kumar ${ }^{\mathrm{a}, \mathrm{b}, *}$ \\ ${ }^{a}$ CSIR-Central Scientific Instruments Organisation, Sector 30C, Chandigarh, Punjab, India \\ ${ }^{b}$ Academy of Scientific and Innovative Research (AcSIR), Ghaziabad, Uttar Pradesh, India \\ [DOI: 10.1117/1.OE.59.12.129802] \\ This article [Opt. Eng. 59(10), 102404 (2019) DOI: 10.1117/1.OE.59.10.102404] was \\ originally published on 12 December 2019 with incorrect information for affiliation "b." \\ The incorrect affiliation information, "Academy of Scientific and Innovative Research, \\ CSIR-Central Scientific Instruments Organisation, Chandigarh, India," has been corrected \\ to "Academy of Scientific \& Innovative Research (AcSIR), Ghaziabad, India." The paper was \\ corrected on 16 December 2020.
}

*Address all correspondence to Raj Kumar, E-mail: raj.optics@csio.res.in 